\title{
Induction of antigen-specific cytotoxic T-cell response by dendritic cells generated from ecto-mesenchymal stem cells infected with an adenovirus containing the MAGE-D4a gene
}

\author{
SHIJIE HU ${ }^{1 *}$, BING LI $^{1 *}$, XUEFENG SHEN ${ }^{2}$, RUI ZHANG ${ }^{3}$, DAKUAN GAO ${ }^{1}$, \\ QINGDONG GUO $^{1}$, YAN JIN ${ }^{4}$ and ZHOU FEI $^{1}$ \\ ${ }^{1}$ Department of Neurosurgery, Xijing Hospital; ${ }^{2}$ Department of Occupational and Environmental Health and \\ The Ministry of Education Key Laboratory of Hazard Assessment and Control in Special Operational Environment, \\ School of Public Health; ${ }^{3}$ Department of Biochemistry and Molecular Biology; \\ ${ }^{4}$ Department of Oral Histology and Pathology, Research and Development Center for Tissue Engineering, \\ School of Stomatology, Fourth Military Medical University, Xi'an, Shaanxi 710032, P.R. China
}

Received March 1, 2015; Accepted January 11, 2016

DOI: $10.3892 / \mathrm{ol} .2016 .4306$

\begin{abstract}
The present study aimed to investigate the feasibility of using ecto-mesenchymal stem cell (EMSC)-derived dendritic cells (DCs) for glioma immunotherapy following infection by a recombinant adenovirus containing the melanoma-associated antigen D4a (MAGE-D4a) gene. The ex vivo cultured EMSCs were infected by the adenoviral plasmid containing MAGE-D4a (pAd/MAGE-D4a). Efficiency of transfection was evaluated through the detection of green fluorescent protein-marked MAGE-D4a. The MAGE-EMSCs were induced to differentiate into DCs, termed as MAGE-EMSCs-DCs. The morphology was subsequently analyzed under a microscope, and methyl thiazolyl tetrazolium (MTT) and interferon- $\gamma($ IFN- $\gamma)$ assays were performed
\end{abstract}

Correspondence to: Dr Zhou Fei, Department of Neurosurgery, Xijing Hospital, Fourth Military Medical University, 15 Changlexilu, Xi'an, Shaanxi 710032, P.R. China

E-mail: hushijie1979@126.com

Professor Yan Jin, Department of Oral Histology and Pathology, Research and Development Center for Tissue Engineering, School of Stomatology, Fourth Military Medical University, 145 Changlexilu, Xi'an, Shaanxi 710032, P.R. China

E-mail: yanjinfmmu@vip.sina.com

*Contributed equally

Abbreviations: EMSCs, ecto-mesenchymal stem cells; DC, dendritic cells; IFN- $\gamma$, interferon- $\gamma$; MOI, multiplicity of infection; CNS, central nervous system CNS; GBM, glioblastoma multiforme; MAGE, melanoma antigen; DMEM, Dulbecco's modified Eagle's medium; MACS, magnetic activated cell sorting; LNGFR, low-affinity nerve growth factor receptor; IL-3, interleukin-3; GM-CSF granulocyte-macrophage colony stimulating factor

Key words: ecto-mesenchymal stem cells, dendritic cells, MAGE-D4a, gene therapy, immunotherapy to analyze the cytotoxicity of the MAGE-EMSC-DCs on the human glioma U251 cell line. Following purification by magnetic-activated cell sorting, the EMSCs grew into swirls, with a long spindle shape and were fibroblast-like. The gene transfected with recombinant adenovirus vectors maintained high and stable expression levels of MAGE-D4a, and its efficiency was increased in a multiplicity of infection-dependent manner. The results of the MTT assay indicated that the T cells, primed by the recombinant MAGE-D4a-infected EMSC-DCs in vitro, recognized MAGE-D4a-expressing tumor cell lines in a human leukocyte antigen class I-restricted manner, and evoked a higher cytotoxic T cell (CTL) response. The CTL response induced by the MAGE-EMSC-DCs, co-cultured with the U251 cells for $24 \mathrm{~h}$, produced $765.0 \mathrm{pg} / \mathrm{ml}$ IFN- $\gamma$, which was significantly greater when compared to the control wells. T lymphocytes stimulated by MAGE-EMSC-DCs evoke a higher CTL response to human glioma cell lines, and may serve as a promising therapeutic modality for the treatment of MAGE-D4a-expressing glioma.

\section{Introduction}

Glioma is the most common neoplasm of the central nervous system (CNS). Glioblastoma multiforme (GBM) is one subtype of glioma, presenting with high-grade malignancy, high rates of disease recurrence and a poor patient prognosis. Despite improvements in radiation therapy delivery, systemic cytotoxic therapy options and surgical techniques, the median survival time for patients with newly-diagnosed GBM subsequent to trimodality therapy is 15 months (1). Thus, patients with GBM require comprehensive treatment to achieve a positive therapeutic outcome $(2,3)$.

A number of active and passive immunotherapy approaches that are being developed have demonstrated potential to improve the survival time of patients with GBM, whilst exerting minimal severe side effects (4). Research regarding brain tumor immunology has resulted in the development of novel immunotherapy strategies, including cytotoxic T-lymphocyte therapies 
and dendritic cell (DC) vaccines (5,6). In pediatric patients with glioma, DC-based immunotherapies may exert certain clinical benefits, particularly in individuals with minimal residual disease; however, additional investigation of such treatment is necessary (7-9).

Unconventional treatment options, including gene therapy combined with immunocyte-based immunotherapy, also offer potential therapeutic approaches to reduce glioma-associated mortality (10). Gene therapies have been implicated in the treatment of brain tumors, with animal models demonstrating preclinical efficacy, in addition to encouraging safety profiles observed in phase I clinical trials; however, in phase III clinical trials, such therapies have thus far failed to demonstrate significant therapeutic efficacy $(11,12)$.

The melanoma-associated antigen (MAGE) family consists of 10 subfamilies, with 1-15 genes in each one $(13,14)$. MAGE-D4a is expressed in a large percentage of human CNS tumors, indicating that it may serve as a potential target for immunotherapy treatment $(15,16)$. In the present study, ecto-mesenchymal stem cells (EMSCs) were isolated and cultured, and subsequently infected with an adenoviral plasmid containing MAGE-D4a (pAd/MAGE-D4a). The MAGE-EMSCs were then induced to differentiate into DCs, and their cytotoxicity on the human glioma U251 cell line was analyzed.

\section{Materials and methods}

Isolation and culture of human EMSCs. A human embryo (50 days of pregnancy) recovered from a spontaneous abortion, initiated by trauma, was donated for tissue isolation. The maxillary and mandibular processes of the embryo were dissected and divided into $<1 \mathrm{~mm}$ sections. The sections were then incubated in a $0.025 \%$ trypsin solution (Gibco; Thermo Fisher Scientific, Inc., Waltham, MA, USA) at $37^{\circ} \mathrm{C}$ for $10 \mathrm{~min}$. The solution was blown slightly to scatter the pieces into a single cell suspension. Enzyme digestion was ceased by neutralization with the equal volume of fresh Dulbecco's modified Eagle's medium (DMEM; Gibco; Thermo Fisher Scientific, Inc.) containing $10 \%$ fetal bovine serum (FBS; Gibco; Thermo Fisher Scientific, Inc.). The cell suspension was centrifuged at $800 \mathrm{rpm}(160 \mathrm{xg})$ for $6 \mathrm{~min}$. The cell pellet was resuspended by DMEM-F12 containing leukemia inhibitory factor (LIF), filtered through a $75-\mu \mathrm{m}$ steel mesh screen to remove cell aggregates and undigested tissues, and was plated in DMEM into tissue culture flasks (Corning Incorporated, Corning, NY, USA). The cells were maintained at $37^{\circ} \mathrm{C}$ in $5 \%$ $\mathrm{CO}_{2}$ for $40 \mathrm{~min}$. The majority of the cells were observed to be adherent under an inverted microscope (CK40; Olympus Corportion, Tokyo, Japan). The culture medium was then switched to DMEM-F12 containing LIF, and 2-3 days later the cells were analyzed under the inverted microscope and were subsequently resuspended for magnetic-activated cell sorting (MACS).

Primary human EMSC (hEMSC) purification by MACS. The present study utilized the indirect immune MACS method to purify the hEMSCs. The magnetic beads were washed, thoroughly, oscillated to achieve sufficient mixing and transferred to a test tube. Following 2 min of standing, the supernatant was discarded. Phosphate-buffered saline (PBS) containing 0.1\% calf serum (Qing Lake Calves, JinHua, China) was added to repeat the aforementioned washing procedure. Following three washes, the magnetic beads were resuspended and the hEMSCs were resuspended in PBS containing $0.1 \%$ serum. Low-affinity nerve growth factor receptor (LNGFR) rabbit anti-rat polyclonal antibody (dilution, 1:200; catalog no., sc-8317; Santa Cruz Biotechnology, Inc., Dallas, TX, USA), using a kit according to the manufacturer's protocols (Dynal Biotech, Carlsbad, CA, USA) was then added into the resuspended solution and incubated at $4^{\circ} \mathrm{C}$ for $30 \mathrm{~min}$. PBS containing $0.1 \%$ serum was used to wash and wipe off the residual antibody. The cell suspensions were incubated with magnetic beads at $4^{\circ} \mathrm{C}$ for 6 min at a concentration of $1 \times 10^{11}$ beads $/ \mathrm{ml}$ cell suspension. The tube was held stirring on a magnetic rack for $2 \mathrm{~min}$, with the supernatant subsequently discarded. PBS containing $0.1 \%$ serum was applied to resuspend and wash the cells twice. Subsequent to the supernatant being discarded, the magnetic bead separation fluid $(0.2 \mathrm{~mol} / 1$ sodium citrate; $\mathrm{pH} 2)$ was added and lightly shaken to blend for $2 \mathrm{~min}$. Following $2 \mathrm{~min}$ of stewing, the supernatant was collected. The procedures were repeated several times to discard the residual magnetic beads, and the collected supernatant was incubated to expand the cell population. The growth and morphology of the cells were observed under an inverted microscope every 2-3 days. The third passage of hEMSCs were obtained, centrifuged, washed and fixed by glutaraldehyde. The prepared ultrathin sections were observed under a transmission electron microscope (JEM-2000EX; JEOL, Ltd., Tokyo, Japan).

Construction of the recombinant Ad-MAGE-D4a. The MAGE-D4a sequence was obtained by reverse RNA transcription of the human glioma U251 cell line (Type Culture Collection of the Chinese Academy of Sciences, Shanghai, China), and was inserted into the adenoviral shuttle vector-pTracer-green fluorescent protein (GFP; Agilent Technologies, Inc., Santa Clara, CA, USA). MAGE-D4a DNA was linearized with PmeI and co-transformed with the pAdEasy-1 Vector (cat no. 240005; Agilent Technologies, Inc.) into the competent bacterial strain BJ5183, which facilitates efficient recombination. Following screening, recombinants for the adenoviruses, Ad/MAGE-D4a and Ad-GFP (containing no insert sequence as a control), were generated. The recombinant DNA was identified by the restriction endonuclease PacI and transfected into human embryonic kidney (HEK)293 cells subsequent to linearization. The cells were collected once cytopathogenic effects had appeared, and the generated recombinant adenoviruses were isolated. Expression of the MAGE-D4a gene was detected by polymerase chain reaction (PCR) using a PicoTiterPlate ${ }^{\mathrm{TM}}$. Briefly, the DNA amplification procedure was performed on an extremely small platform in picolitre quantities. Subsequently the products were available to be transferred to solid supports for transcription, translation or sequencing (17), which were performed by AuGCT (Beijing, China). The virus titer was measured on day 14 post-infection. The primers used were as follows: MAGE-D4a forward, 5'-ACGCGGTACCCATGGCTGAGGGAAGCTTCAGCG TGC-3' and reverse, 5'-GGCTCGAGACGGTGCTGGATC CAGGAGAAGAAG-3' (Augct DNA-Syn Biotechnology Co., Ltd., Beijing, China). 
pAd/MAGE-D4a adenoviral infection of the hEMSCs. The sixth passage hEMSCs following MACS were seeded in a 6 -well plate at a concentration of $2 \times 10^{6}$ cells per well. The cells were transduced with pAd/MAGE-D4a once they had achieved $\sim 80 \%$ confluency. Infection was performed with a multiplicity of infection (MOI) of $0.1,1,10$ and 100 plaque-forming units/cell in $1 \mathrm{ml}$ infection buffer at room temperature for $60 \mathrm{~min}$. Subsequent to infection, the hEMSCs were incubated for the indicated time periods at $37^{\circ} \mathrm{C}$ with $5 \% \mathrm{CO}_{2}$ in a humidified atmosphere. Following a total of $48 \mathrm{~h}, \mathrm{pAd} / \mathrm{MAGE}-\mathrm{D} 4 \mathrm{a}$ expression was analyzed by identifying the $\mathrm{GFP}^{+}$cells under a fluorescence microscope (IX71; Olympus Corporation). In addition, total RNA was extracted from pAd/MAGE-D4a adenoviral infected hEMSCs using TRIzol reagent (Invitrogen; Thermo Fisher Scientific, Inc.), according to the manufacturer's protocol, in order to check the expression of MAGE-D4a. RNA $(1 \mu \mathrm{g})$ was used to perform reverse transcription (RT) to provide complementary DNA using the PrimeScript RT Master Mix (Perfect Real Time; Takara Biotechnology Co., Ltd., Dalian, China). For the quantitative polymerase chain reaction (qPCR), $10 \mu 1$ SYBR Green Premix Ex Taq ${ }^{\mathrm{TM}}$ (Takara Biotechnology Co., Ltd.), $1 \mu 1$ primers and $2 \mu \mathrm{l}$ cDNA were used. The primer sequences were as follows and were obtained from Beijing AuGCT Biotechnology Co., Ltd. (Beijing, China): MAGE-D4a, forward 5'-CCAGCTTCTTCTCCTGGATC-3 and reverse 5'-GTAACACTGATACCCAAAACATG-3'. Glyceraldehyde 3-phosphate dehydrogenase was employed as a control, with the primer sequence as follows: forward, 5'-TCACCAGGGCTGCTTTTAAC-3'; and reverse, 5'-GACAAGCTTCCCGTTCTCAG-3'. PCR was performed on a C1000 Touch ${ }^{\mathrm{TM}}$ Thermal Cycler (Bio-Rad Laboratories, Inc., Hercules, CA, USA), and the cycling conditions were as follows: Initial denaturation at $95^{\circ} \mathrm{C}$ for $10 \mathrm{~min}$, followed by 40 cycles of $15 \mathrm{sec}$ at $95^{\circ} \mathrm{C}$ and $1 \mathrm{~min}$ at $60^{\circ} \mathrm{C}$. Each cDNA sample was run three times. MAGE-D4a mRNA expression was normalized to glyceraldehyde 3-phosphate dehydrogenase and fold differences were determined using the $2^{-\Delta \Delta \mathrm{Cq}}$ method (18).

Induction of differentiation of the pAd/MAGE-D4a-hEMSCs to DCs. Subsequent to screening, the $\mathrm{GFP}^{+}$hEMSCs were counted at a concentration of $1 \times 10^{4}$ cells $/ \mathrm{ml}$. To distinguish whether the infection was successful, $\mathrm{GFP}^{+}$cells were determined by making comparisons between green fluorescence and white light. Recombinant human granulocyte-macrophage colony stimulating factor (GM-CSF; $100 \mathrm{ng} / \mathrm{ml}$; Sigma-Aldrich, St. Louis, MO, USA) and recombinant human interleukin-3 (IL-3; $200 \mathrm{U} / \mathrm{ml}$; Sigma-Aldrich) were added to the culture medium for 17 days. During this time, the medium was changed for half dose with the combination of the above factors every 3 days. On day 19, recombinant human tumor necrosis factor (200 U/ml; Sigma-Aldrich) was added to continue the induction. The cell morphology was subsequently analyzed under a fluorescence microscope.

Ex vivo cytotoxic $T$ cell $(C T L)$ response. The human effector cell culture was formed using peripheral blood mononuclear cells that had been separated using the Ficoll-Hypaque density gradient centrifugation method and resuspended in RPMI 1640 containing $10 \%$ calf bovine serum. The cells were then seeded in a 6-well plate at a concentration of $5 \times 10^{6}$ cells per well and incubated at $37^{\circ} \mathrm{C}$ containing $5 \% \mathrm{CO}_{2}$ in a humidified atmosphere for $2 \mathrm{~h}$. The non-adherent cells were collected, counted at a concentration of $1 \times 10^{6}$ cells $/ \mathrm{ml}$ and cultured at $37^{\circ} \mathrm{C}$ in a humidified atmosphere containing $5 \%$ $\mathrm{CO}_{2}$. The cells were centrifuged every 4 days, in addition to changing the culture medium. The cells were mixed with the pAd/MAGE-D4a-hEMSC-induced DCs at a proportion of 5:1.

Groups. The three groups of cells utilized were as follows: Group 1,pAd/MAGE-D4a-hEMSC-induced DCs with effector lymphocytes, termed MAGE-hEMSCs-DC + lymphocytes (MAGE-EMSCs-DC-L); Group 2, hEMSCs transduced with pAd/MAGE-D4a with effector lymphocytes, termed MAGE-hEMSCs + lymphocytes (MAGE-EMSCs-L); and Group 3, lymphocytes (L).

CTL response assay. A methyl thiazolyl tetrazolium (MTT) assay was performed to analyze the CTL response. The effector cells (MAGE-EMSCs-DC-L, MAGE-EMSCs-L and L) and target cells (human glioma U251 cell line) were mixed and seeded in a 96-well plate at a ratio of 10:1 (effector cells, $10^{5}$ cells per well; target cells, $10^{4}$ cells per well). The effector cells and target cells were respectively seeded alone to serve as blank controls. There were 8 samples within each group. Cells of each groups were cultured for $24 \mathrm{~h}$ at $37^{\circ} \mathrm{C}$ in a humidified atmosphere with $5 \% \mathrm{CO}_{2}$. Following the MTT reaction, the absorbance (A) of each wells was measured at $490 \mathrm{~nm}$. The formula for the cytotoxicity index (CTI) was as follows: CTI $=[1-($ trial hole A - effector cells A) / (target ce lls A)] x100.

Interferon- $\gamma(I F N-\gamma)$ assay by enzyme-linked immunosorbent assay (ELISA). The MAGE-EMSC-DCs, or the normal control DC, was incubated for 8 days in 10\% FBS RPMI 1640 medium in a $5 \% \mathrm{CO}_{2}$ incubator. The MAGE-EMSC-DCs, GFP-EMSC-DCs or normal control DCs were then co-incubated with the U251 cell line CTLs at a ratio of 20:1 for $24 \mathrm{~h}$. The cell culturing supernatant was harvested, and ELISA was utilized to determine IFN- $\gamma$ levels using the human IFN- $\gamma$ and biotinylated human IFN- $\gamma$ detection antibodies, according to the Human IFN- $\gamma$ ELISA kit (catalog no., RAB0222; Sigma-Aldrich).

Statistical analysis. The data were expressed as means and standard deviations. The ex vivo CTL response analyses and IFN- $\gamma$ assay were performed according to the Student-Newman-Keuls test. $\mathrm{P}<0.05$ was considered to indicate a statistically significant difference. All the data were analyzed using SPSS software, version 18.0 (SPSS, Inc., Chicago, IL, USA).

\section{Results}

Characterization of the hEMSCs. Following MACS, the majority of $\mathrm{LNGFR}^{+}$cells post-culture $\left(37^{\circ} \mathrm{C}\right.$ in a humidified atmosphere containing $5 \% \mathrm{CO}_{2}$ ) were attached, and demonstrated a fibroblast-like morphology in the third passage (Fig. 1A and B). The ultrastructure of the hEMSCs was characterized by mesenchymal-like cells aggregated in an irregular shape. The nucleolus was marked and close to the 

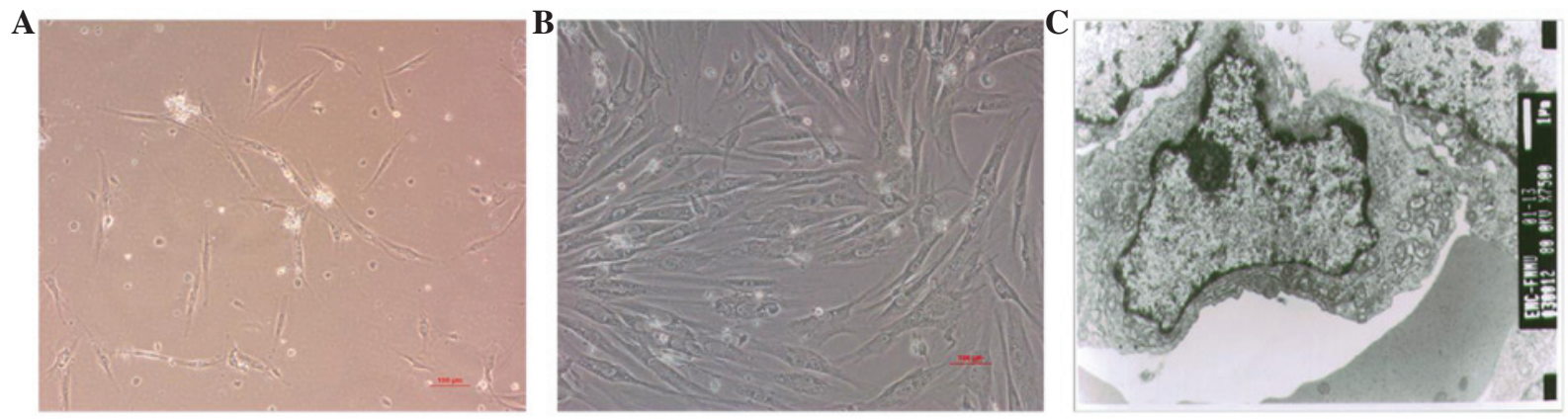

Figure 1. Characterization of the hEMSCs. (A) Morphology of the $\mathrm{LNGFR}^{+}$cells following culture for $12 \mathrm{~h}$ (magnification, $\mathrm{x} 100$ ); (B) Morphology of the $\mathrm{LNGFR}^{+}$cells passaged to the third generation (magnification, x100). (C) Ultrastructure of the hEMSCs (magnification, x7500). hEMSCs, human ecto-mesenchymal stem cells; LNGFR, low-affinity nerve growth factor receptor.
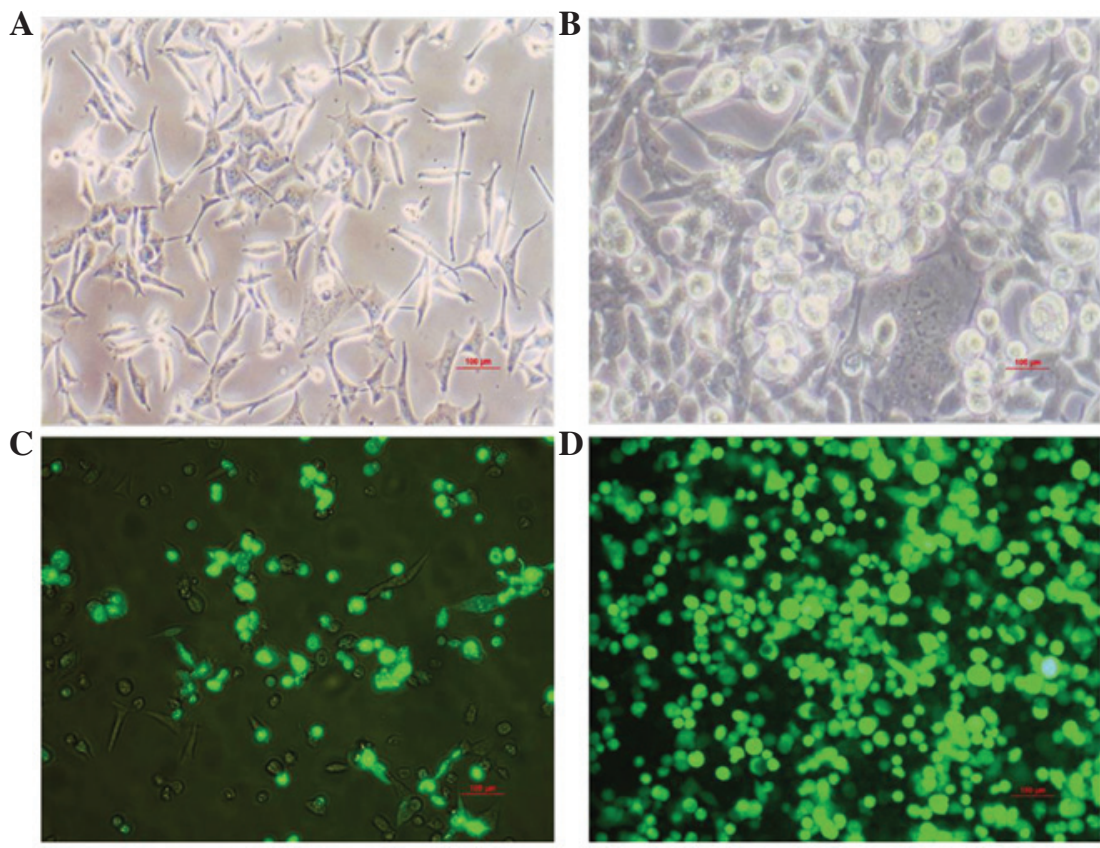

Figure 2. (A) Normal HEK293. (B) Following 3-10 days of infection by pAd/MAGE-D4a, the infected cells demonstrated characteristic cytopathogenic effects. The cells were initially round in shape, and once the refractivity had increased, they then detached from the bottom of the culture bottle and formed grape-like aggregates. (C) GFP expression level was weak during the first round of amplification. (D) GFP expression level had significantly increased by the fourth round of amplification. Magnification, x100. GFP, green fluorescence protein; MAGE-D4a, melanoma antigen family D4a.

cell membrane, and contained a large ratio of nucleuic to cytoplasmic material. There was a greater amount of euchromatin than heterochromatin within the cells, a large number of mitochondria and a small amount of rough endoplasmic reticulum and ribosomes, which indicated the undifferentiation and high proliferation activity of the hEMSCs (Fig. 1C).

Transgene expression in vitro. To identify whether there was an increase in MAGE-D4a expression, the total and MAGE-D4a RNA were subjected to agarose gel electrophoresis. It was identified that the intensity of the $28 \mathrm{~S}$ band was much more potent than that of the $18 \mathrm{~S}$ band, whilst no band was detected at $5 \mathrm{~S}$; therefore demonstrating that the integrity of the total RNA was marked. RNA A260/280 was 2.00, indicating that it was suitable for RT-PCR.

Construction ofT-easy/MAGE-D4a.T vector-linked MAGE was screened through DH-5a, and the smaller clones were picked out.
The plasmids were then extracted following bacteria expansion, identified by $K p n \mathrm{~L} / \mathrm{XhoI}$ double-enzyme cuts. The gel bands obtained through electrophoresis were sent to Shanghai Shenggong Co. Ltd. (Shanghai, China) for sequencing; the results were consistent with those obtained from GenBank (www.ncbi.nlm. nih.gov/genbank), and no mutation was detected.

Construction of pTrack-cmv-GFP. Through enzyme cutting, it was identified that MAGE-D4a was successfully introduced into the pTrack-cmv-GFP plasmid. Lanes 1 to 6 were multiple plasmids extracted from positive clones, and were subjected to KpnL/Xhol double-enzyme cutting.

Infection of the HEK293 cells. Monolayer HEK293 cells were infected by pAd/MAGE-D4a. The infected cells demonstrated characteristic cytopathogenic effects, initially being round in shape, and once refractivity had increased, they detached from the bottom of the culture bottle and formed grape-like 


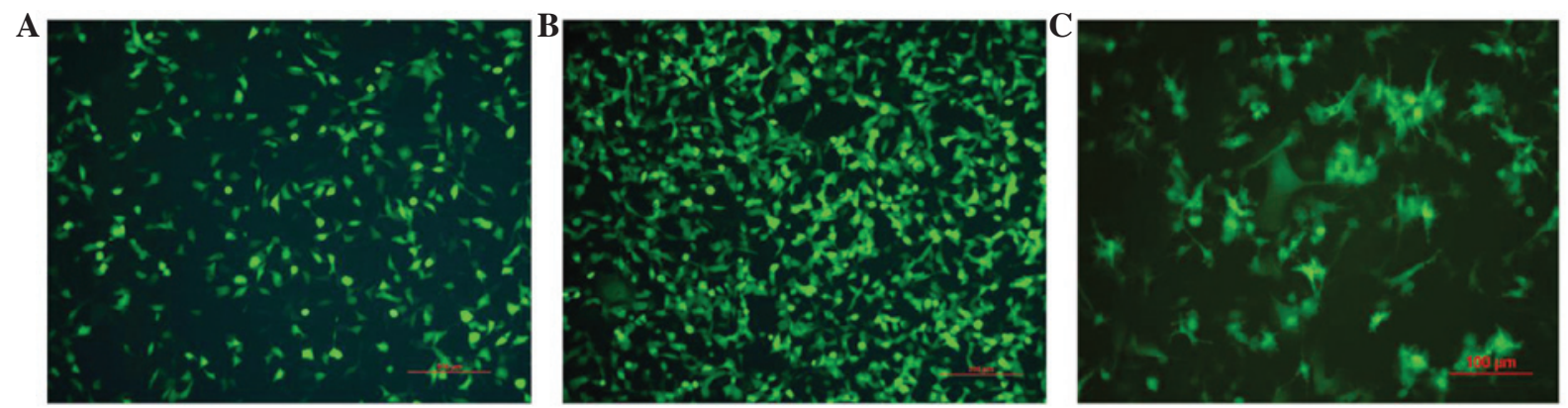

Figure 3. pAd/MAGE-D4a adenoviral infection of the hEMSCs: (A) MOI of 10 (magnification, x40); (B) MOI of 100 (magnification, x40). (C) pAd/MAGE-D4a-hEMSC-induced DCs exhibited veiled-dendritic morphology typical of DCs; the pricking and dendritic eminences on the cells surface were also observed. (magnification, x200). MAGE-D4a, melanoma antigen family D4a; hEMSCs; human ecto-mesenchymal stem cells; MOI, multiplicity of infection; DCs, dendritic cells.

A

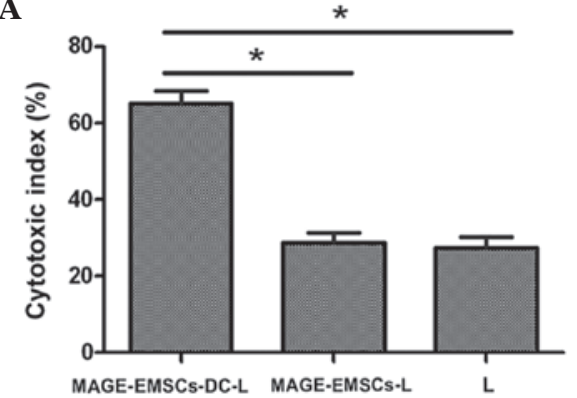

B

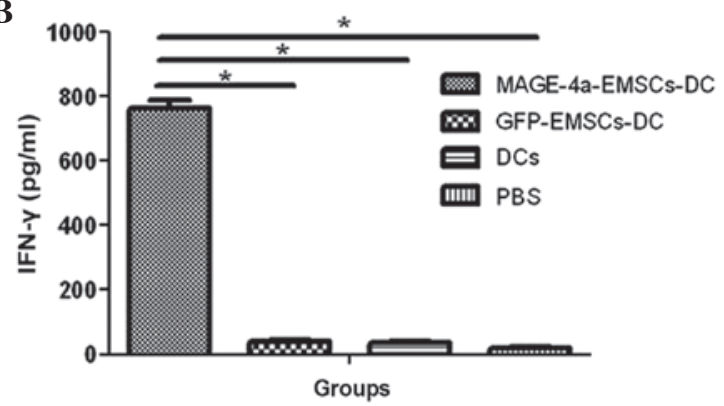

Figure 4. (A) Cytotoxicity indexes in the MAGE-EMSC-DC-L, MAGE-EMSC-L and L groups were 65.1 $\pm 3.4,28.8 \pm 2.55$ and $27.4 \pm 2.89 \%$, respectively. The cytotoxic index was significantly greater in the MAGE-EMSC-DC-L group compared with the other two groups. Error bars represent the mean \pm SD from the three groups. (B) IFN- $\gamma$ release in the culture supernatants of the MAGE-4a specific CTLs that were exposed to the MAGE-EMSC-DCs, GFP-EMSC-DCs or the normal control DCs at a ratio of 20:1 (MOI, 100). The CTLs induced by the MAGE-EMSC-DCs, co-cultured with the U251 cells for 24 h, produced $765.0 \mathrm{pg} / \mathrm{ml}$ IFN- $\gamma$, which was much higher compared with the control wells. Error bars represent the mean \pm SD from the three groups. * $<0.05$. MAGE, melanoma antigen family; EMSCs; ecto-mesenchymal stem cells; DC, dendritic cell; SD, standard deviation; IFN, interferon; CTLs, cytotoxic T-cell; MOI, multiplicity of infection; GFP, green fluorescence protein; PBS, phosphate buffered-saline.

aggregates (Fig. 2A and B). Protein amplification was analyzed by detecting GFP expression. The GFP expression level had significantly increased by the fourth round of amplification (Fig. 2C and D).

RT-PCR. An electrophoresis band at 2,200 bp was amplified, indicating that the virus contained the target gene. Under the same conditions, there was no band detected in either the control or the empty virus groups.

pAd/MAGE-D4a Titer measurement and adenoviral infection of the hEMSCs. On day 14 post-infection, 10-100 wells of cells were counted, and the virus titer was measured at $2 \times 10^{8} \mathrm{pfu} / \mathrm{ml}$. Once the hEMSCs had been infected by the pAd/MAGE-D4a with a MOI of 10, $\sim 50 \%$ of the hEMSCs were $\mathrm{GFP}^{+}$(Fig. 3A). When the hEMSCs were infected by the pAd/MAGE-D4a with a MOI of $100, \sim 80 \%$ of hEMSCs were $\mathrm{GFP}^{+}$, thus demonstrating that high biological activity functions as a gene transfer vector (Fig. 3B).

Morphological features of the pAd/MAGE-D4a-hEMSC derived DCs. The induced cells from the hEMSCs exhibited a veiled dendritic morphology typical of DCs. The pricking and dendritic eminences on the cell surfaces were observed under the microscope (Fig. 3C).
Ex vivo CTL response. The MTT assay demonstrated that the cytotoxity index in the MAGE-EMSC-DC-L, MAGE-EMSC-L and $\mathrm{L}$ groups were $65.1 \pm 3.4,28.8 \pm 2.55$ and $27.4 \pm 2.89 \%$, respectively. The cytotoxic index was significantly higher in the MAGE-EMSCs-DC-L group compared with the other two groups $(\mathrm{P}<0.05$; Fig. 4A).

IFN- $\gamma$ assay with ELISA. IFN- $\gamma$ release in the culture supernatants of specific CTLs was significantly higher in the wells of the co-culture with MAGE-4a-EMSC-DCs $(765.0 \mathrm{pg} / \mathrm{ml})$ compared with the wells containing control DCs $(\mathrm{P}<0.05$; Fig. 4B); indicating that the MAGE gene may be recognized by CTLs.

\section{Discussion}

Of all malignant primary brain tumors, GBM is the most prevalent and the most lethal. Over the years, researchers in basic and clinical studies have aimed to improve the survival rate for patients with GBM; however, the prognosis remains to be poor (19). Preclinical data and active immunotherapy studies have concentrated on synthetic antigen vaccination and tumor lysate strategies (20). Marsh et al (1) reviewed the existing clinical data regarding immunological therapy in the treatment of GBM and identified that the existing phase I and II data suggest that such therapies may produce 
valid, and sometimes durable, responses in patients with GBM with mild toxicity compared with other existing therapies. The results of the present study demonstrate that DCs derived from hEMSCs expressing MAGE-D4 can induce specific CTL responses against $\mathrm{U} 251$ cells in vitro.

In the current study, hEMSCs were isolated and cultured and then purified and expanded ex vivo using the indirect immune MACS method. Cranial neural crest-derived hEMSCs differentiate into a number of different cell types, including peripheral nervous system glial cells, neurons, melanocytes and certain paraendocrine and endocrine cells (21). Furthermore, the pluripotency of hEMSCs presents the opportunity to obtain DCs and/or microglia in vitro and in vivo (22). The current study subsequently constructed recombinant Ad-MAGE-D4a, which was used as a target (or tool) in the MAGE-D4a gene, and has also been utilized in immunotherapy for malignant glioma and other tumors $(23,24)$. The purified hEMSCs were infected by Ad-MAGE-D4a and induced into DCs. Under the GM-CSF and IL-3 culture condition, the MAGE-EMSC-DCs exhibited typical DC morphology with elongated dendritic processes, which upregulated the expression of major histocompatibility complex II, cluster of differentiation (CD)11c, CD86, CD80 and CD40, and downregulated the expression of LNGFR (22). DCs are antigen presenting cells, and currently DC-based vaccinations, which provoke host immune responses against tumor antigens, are being considered as promising immunotherapeutic agents $(25,26)$.

In the present study, ex vivo CTL response analysis demonstrated that the cytotoxic index of MAGE-EMSC-DC-L was significantly higher than the indexes of the other two groups, which indicated that a potent and specific antitumor immune response may be induced by the MAGE-EMSC-DC vaccine. The CTLs induced by the MAGE-EMSC-DCs, co-cultured with the U251 cells for $24 \mathrm{~h}$, produced greater IFN $-\gamma$ release compared with the control groups. Interferon- $\gamma$ is a cytokine that interacts with cell-surface receptors, subsequently activating the transcription of genes. It is considered that the genes activated offer therapeutic potential by inhibiting tumor angiogenesis, disrupting proliferative mechanisms and increasing tumor immunogenicity (27).

Despite the notable results from the present study, there are certain issues that require clarification. Firstly, cell apoptosis was not analyzed with flow cytometry following the construction of pAd/MAGE-D4a; the present study instead depended on GFP fluorescence post-infection, which detected that the pAd/MAGE-D4a infection had no significant effect on the apoptosis of the hEMSCs when compared with the mock-infected pAd/MAGE-D4a. Secondly, no data from animal experiments was utilized to demonstrate that the MAGE-D4-EMSCs could differentiate into microglial cells in the CNS and serve as antiglioma effectors. Therefore, further investigation is required to assess the effectiveness and safety of the application of MAGE-EMSC-DCs to induce antigen-specific CTL responses in vivo.

Recurrence of GBM primarily occurs due to the tumor itself developing several mechanisms that allow it to escape the immune system (28). Thus, gene and immunotherapy are currently the most promising strategies to solve this problem. The results of the present study have demonstrated that EMSCs may be effectively infected by adenovirus vectors to express MAGE-D4a. MAGE-D4-EMSCs differentiate into DCs, which subsequently induce MAGE-D4a-specific CTL responses against tumor cells in vitro. Based on the results from the current study, MAGE-D4-EMSC-DCs may serve as a feasible and effective approach for the treatment of glioma and other CNS tumors. Additional immunotherapy strategies are currently in development for the treatment of GBM, and future studies may consider the application of combinatorial gene and immunotherapy strategies in preclinical trials.

\section{Acknowledgements}

The present study was supported by the Youth Science Fund Project of the National Natural Science Foundation of China (grant no. 81101710).

\section{References}

1. Marsh JC, Goldfarb J, Shafman TD and Diaz AZ: Current status of immunotherapy and gene therapy for high-grade gliomas. Cancer Control 20: 43-48, 2013.

2. Chen $\mathrm{J}$ and $\mathrm{Xu} \mathrm{T}$ : Recent therapeutic advances and insights of recurrent glioblastomamultiforme.FrontBiosci(LandmarkEd) 18: 676-684, 2013.

3. Ajeawung NF, Wang HY and Kamnasaran D: Progress from clinical trials and emerging non-conventional therapies for the treatment of Medulloblastomas. Cancer Lett 330: 130-140, 2013.

4. Xu Q, Yuan X and Yu JS: Glioma stem cell research for the development of immunotherapy. Adv Exp Med Biol 746: 216-225, 2012.

5. Daga A, Bottino C, Castriconi R, Gangemi R and Ferrini S: New perspectives in glioma immunotherapy. Curr Pharm Des 17: 2439-2467, 2011.

6. Petrosiute A, Auletta JJ and Lazarus HM: Achieving graft-versus-tumor effect in brain tumor patients: From autologous progenitor cell transplant to active immunotherapy. Immunotherapy 4: 1139-1151, 2012.

7. Ardon H, Van Gool SW, Verschuere T, Maes W, Fieuws S, Sciot R, Wilms G, Demaerel P, Goffin J, Van Calenbergh F, et al: Integration of autologous dendritic cell-based immunotherapy in the standard of care treatment for patients with newly diagnosed glioblastoma: Results of the HGG-2006 phase I/II trial. Cancer Immunol Immunother 61: 2033-2044, 2012.

8. Prins RM, Wang X, Soto H, Young E, Lisiero DN, Fong B, Everson R, Yong WH, Lai A, Li G, et al: Comparison of glioma-associated antigen peptide-loaded versus autologous tumor lysate-loaded dendritic cell vaccination in malignant glioma patients. J Immunother 36: 152-157, 2013.

9. Lasky JL III, Panosyan EH, Plant A, Davidson T, Yong WH, Prins RM, Liau LM and Moore TB: Autologous tumor lysate-pulsed dendritic cell immunotherapy for pediatric patients with newly diagnosed or recurrent high-grade gliomas. Anticancer Res 33: 2047-2056, 2013.

10. Mineharu Y, Muhammad AK, Yagiz K, Candolfi M, Kroeger KM, Xiong W, Puntel M, Liu C, Levy E, Lugo C, et al: Gene therapy-mediated reprogramming tumor infiltrating $\mathrm{T}$ cells using IL-2 and inhibiting NF- $\mathrm{kB}$ signaling improves the efficacy of immunotherapy in a brain cancer model. Neurotherapeutics 9 : 827-843, 2012.

11. Tobias A, Ahmed A, Moon KS and Lesniak MS: The art of gene therapy for glioma: A review of the challenging road to the bedside. J Neurol Neurosurg Psychiatry 84: 213-222, 2013.

12. Assi H, Candolfi M, Baker G, Mineharu Y, Lowenstein PR and Castro MG: Gene therapy for brain tumors: Basic developments and clinical implementation. Neurosci Lett 527: 71-77, 2012.

13. van der Bruggen $\mathrm{P}$, Traversari $\mathrm{C}$, Chomez $\mathrm{P}$, Lurquin $\mathrm{C}$, De Plaen E, Van den Eynde B, Knuth A and Boon T: A gene encoding an antigen recognized by cytolytic $\mathrm{T}$ lymphocytes on a human melanoma. Science 254: 1643-1647, 1991.

14. Chomez P, De Backer O, Bertrand M, De Plaen E, Boon T and Lucas S: An overview of the MAGE gene family with the identification of all human members of the family. Cancer Res 61: 5544-5551, 2001 
15. Ito S, Kawano Y, Katakura H, Takenaka K, Adachi M, Sasaki M, Shimizu K, Ikenaka K, Wada $\mathrm{H}$ and Tanaka F: Expression of MAGE-D4, a novel MAGE family antigen, is correlated with tumor-cell proliferation of non-small cell lung cancer. Lung Cancer 51: 79-88, 2006.

16. Lee MH, Son EI, Kim E, Kim IS, Yim MB and Kim SP: Expression of cancer-testis genes in brain tumors. J Korean Neurosurg Soc 43: 190-193, 2008.

17. Leamon JH, Lee WL, Tartaro KR, Lanza JR, Sarkis GJ, deWinter AD, Berka J, Weiner M, Rothberg JM and Lohman KL: A massively parallel PicoTiterPlate based platform for discrete picoliter-scale polymerase chain reactions. Electrophoresis 25: 1176,2004

18. Livak KJ and Schmittgen TD: Analysis of relative gene expression data using real-time quantitative PCR and the 2(-Delta Delta C(T)) Method. Methods 25: 402-408, 2001.

19. Zhang X, Zhang W, Mao XG, Zhen HN, Cao WD and Hu SJ: Targeting role of glioma stem cells for glioblastoma multiforme. Curr Med Chem 20: 1974-1984, 2013.

20. Reardon DA, Wucherpfennig KW, Freeman G, Wu CJ, Chiocca EA, Wen PY, Curry WT Jr, Mitchell DA, Fecci PE, Sampson JH and Dranoff G: An update on vaccine therapy and other immunotherapeutic approaches for glioblastoma. Expert Rev Vaccines 12: 597-615, 2013.

21. Chai Y, Jiang X, Ito Y, Bringas P Jr, Han J, Rowitch DH, Soriano P, McMahon AP and Sucov HM: Fate of the mammalian cranial neural crest during tooth and mandibular morphogenesis. Development 127: 1671-1679, 2000.
22. Hu S, Shen X, Zhang R, Zhang Y, Zhang R, Zhang W, Deng Z, Cao Y, Zhou Z, Chen J, et al: Development of rat antigen-presenting cells from pluripotent ecto-mesenchymal stem cells in vitro and in vivo. Mol Immunol 45: 3818-3826, 2008.

23. Syed ON, Mandigo CE, Killory BD, Canoll P and Bruce JN: Cancer-testis and melanocyte-differentiation antigen expression in malignant glioma and meningioma. J Clin Neurosci 19: 1016-1021, 2012.

24. Weeraratne SD, Amani V, Neiss A, Teider N, Scott DK, Pomeroy SL and Cho YJ: miR-34a confers chemosensitivity through modulation of MAGE-A and p53 in medulloblastoma. Neuro-oncol 13: 165-175, 2011.

25. Yang JY, Cao DY, Liu WC, Zhang HM, Teng ZH and Ren J: Dendritic cell generated from CD34+ hematopoietic progenitors can be transfected with adenovirus containing gene of $\mathrm{HBsAg}$ and induce antigen-specific cytotoxic $\mathrm{T}$ cell responses. Cell Immunol 240: 14-21, 2006.

26. Candolfi M, King GD, Yagiz K, Curtin JF, Mineharu Y, Muhammad AK, Foulad D, Kroeger KM, Barnett N, Josien R, et al: Plasmacytoid dendritic cells in the tumor microenvironment: Immune targets for glioma therapeutics. Neoplasia 14: 757-770, 2012.

27. Kane A and Yang I: Interferon-gamma in brain tumor immunotherapy. Neurosurg Clin N Am 21: 77-86, 2010.

28. Szabo AT and Carpentier AF: Immunotherapy in human glioblastoma. Rev Neurol (Paris) 167: 668-672, 2011. 